Bull. Soc. math. France

131 (1), 2003, p. 123-147

\title{
ALTÉRATIONS ET GROUPE FONDAMENTAL PREMIER À $p$
}

\author{
PAR FABRice Orgogozo
}

\begin{abstract}
RÉSumÉ. - Nous démontrons divers résultats sur le plus grand quotient du groupe fondamental étale premier aux caractéristiques, parmi lesquels la formule de Künneth et l'invariance par changement de corps séparablement clos pour les schémas de type fini sur un corps. Ces énoncés sont déduits de faits généraux sur les images directes de champs, une fois spécialisés au cas des torseurs sous un groupe constant fini d'ordre inversible sur la base. Des résultats analogues pour le groupe fondamental modéré sont également discutés.

Abstract (Alterations and the prime-to-p fundamental group). - Various results are proved relating to the largest quotient of the étale fundamental group prime to the residual characteristics, including the Künneth formula and invariance under change of separably closed field for schemes of finite type over a field. These are derived from general facts about direct images of stacks, once specialized to the case of torsors under a constant finite group of invertible order over the base. Analogous results for the tame fundamental group are discussed as well.
\end{abstract}

\section{Introduction}

Soit $k$ un corps algébriquement clos de caractéristique $p$ et soit $p^{\prime}$ l'ensemble des nombres premiers distincts de $p$. Dans [24], il est démontré que, si $X$ et $Y$

Texte reçu le 25 janvier 2002, accepté le 22 mars 2002

FABrice Orgogozo, École normale supérieure, DMA, 45, rue d'Ulm, F-75230, Paris

Cedex 05 (France) - E-mail : Fabrice.Orgogozo@ens.fr

Url : http://www.dma.ens.fr/ orgogozo

Classification mathématique par sujets (2000). — 14F35, 14A20, 14D05.

Mots clefs. - Groupe fondamental, champs, descente, schémas simpliciaux. 
sont des $k$-schémas de type fini connexes, on a alors

$$
\pi_{1}^{p^{\prime}}\left(X \times_{k} Y\right) \stackrel{\sim}{\longrightarrow} \pi_{1}^{p^{\prime}}(X) \times \pi_{1}^{p^{\prime}}(Y),
$$

sous réserve que l'on dispose de la résolution forte des singularités.

Nous prouvons dans cet article que cette formule est valable sans restriction. Nous démontrons en fait des théorèmes de finitude, propreté cohomologique et locale acyclicité génériques sans hypothèse de résolution. Il s'agit ici d'énoncés sur les ensembles de cohomologie de degré 0 et 1 . Une partie de ces résultats figurent sous forme conditionnelle dans loc. cit. Par ailleurs, les analogues en cohomologie abélienne sont démontrés sans restriction dans [8]. L'ingrédient essentiel de ce travail est le théorème de A.J. de Jong [21] sur les altérations, qui remplace la résolution forte des singularités. Les altérations ont en effet la propriété d'être de descente effective universelle pour la catégorie fibrée des faisceaux étales. Comme dans [24], nous travaillons systématiquement dans la 2-catégorie des champs en groupoïdes, qui est le pendant non commutatif de la catégorie dérivée des faisceaux abéliens.

$\mathrm{Au}$ numéro 2 (resp. 3), nous démontrons le théorème de propreté cohomologique et constructibilité (resp. locale acyclicité) générique. Certaines démonstrations initiales de l'auteur ont été modifiées pour faire profiter au lecteur de l'approche plus conceptuelle d'O. Gabber, qui sera certainement plus utile à l'avenir. Le numéro 4 contient divers énoncés de constance locale générique pour les images directes de champs, ainsi que des applications au groupe fondamental premier à $p$ : locale invariance générique des $\pi_{1}^{p \prime}$ des fibres géométriques d'un morphisme, invariance par changement de corps séparablement clos et formule de Künneth. Nous énonçons des résultats semblables pour les groupes d'homotopie supérieurs. Enfin, le numéro 5, indépendant des précédents, concerne la formule de Künneth et l'invariance par changement de corps algébriquement clos pour le groupe fondamental modéré; nous avons recours ici à des techniques de géométrie logarithmique.

Remerciements. - La forme définitive de cet article doit beaucoup à mes nombreuses discussions avec LuC ILlusie. Par ailleurs, OfER GABBER m'a autorisé à inclure les résultats qui figurent dans une lettre qu'il m'a adressée [11]. Il a aussi eu la gentillesse de me faire profiter de sa relecture attentive d'une version précédente de ce texte $(c f$. [12]). Je les remercie très chaleureusement pour leur aide précieuse. Je tiens à remercier également MICHEL RAYNAUD et ISABELLE VIDAL pour d'utiles conversations concernant le numéro 5.

\section{Propreté cohomologique générique}

Soient $X$ un schéma, et $\mathscr{F}$ un champ (en groupoïdes) sur $X$ (muni de la topologie étale). Dans toute la suite, seuls la topologie étale et les champs en groupoïdes seront envisagés et nous omettrons donc ces qualificatifs.

TOME $131-2003-\mathrm{N}^{\mathrm{O}} 1$ 
Notons $\pi_{0}(\mathscr{F})$ le faisceau associé au préfaisceau

$U / X \longmapsto\{$ classes d'isomorphismes d'objets de $\mathscr{F}(U)\} ;$

c'est aussi le faisceau des sous-gerbes maximales de $\mathscr{F}$.

Soient $U / X$ un ouvert étale et $x_{U} \in \mathrm{Ob} \mathscr{F}(U)$; nous noterons $\pi_{1}\left(\mathscr{F}, x_{U}\right)$ le $U$-faisceau Aut $\left(x_{U}\right)^{(1)}$.

Si $\mathrm{L}$ est un ensemble de nombres premiers, rappelons que le champ $\mathscr{F}$ est ind-L-fini, si pour toute section locale $x$, le faisceau en groupes $\pi_{1}(\mathscr{F}, x)$ est ind-L-fini au sens de $[2,1.5]$. Cela signifie que pour tout point géométrique $\xi$, la fibre de ce faisceau en $\xi$ s'identifie à la colimite filtrante de ses sous-groupes finis de L-torsion. Le champ $\mathscr{F}$ est dit constructible si le faisceau $\pi_{0}(\mathscr{F})$ et les divers $\pi_{1}(\mathscr{F}, x)$ sont constructibles ( $c f$. loc. cit., 2.3 ) ; nous ne considérerons que le cas d'un schéma de base noethérien pour cette dernière notion. Pour tout schéma $X$, nous noterons $\mathrm{L}_{X}$ l'ensemble des nombres premiers inversibles sur $X$. (Dans le cas particulier où seul un nombre premier $p$ n'est pas inversible sur $X$, nous noterons $p^{\prime}$ l'ensemble $\mathrm{L}_{X}$.)

Pour alléger les notations, nous désignerons souvent par la même lettre un $X$-champ et son extension, comme catégorie fibrée, à la catégorie des schémas sur $X$ ( $c f$. [13, VII.2.2.7]). De même, nous noterons souvent par des égalités des flèches qui ne sont en fait que des équivalences.

Pour fixer les notations, et motiver les théorèmes généraux qui vont suivre, voici quelques exemples. Le dernier - le plus important pour nos applications - précise le lien entre variation du groupe fondamental en famille (lié à l'étude des faisceaux non abéliens $\mathrm{R}^{1} f_{*} G$ pour une famille $f: X \rightarrow S$ et $G$ un groupe fini constant) et le formalisme des images directes de champs que nous allons étudier par la suite.

2.1. Exemples. - Soient $f: X \rightarrow Y$ et $g: Z \rightarrow X$ des morphismes de schémas.

Champs de Picard. - À tout complexe $K=\left[d: \mathcal{L}^{-1} \rightarrow \mathcal{L}^{0}\right]$ de faisceaux abéliens sur $X$, on associe un champ $\operatorname{ch}(K)$. C'est le champ associé au préchamp dont le préfaisceau des objets est $\mathcal{L}^{0}$, et dont les homomorphismes entre deux sections locales $a$ et $b$ sur $U$ sont les sections $s \in \mathcal{L}^{-1}(U)$ telles que $\mathrm{d} s=b-a$. Cette construction, qui se factorise par $\mathrm{D}^{[-1,0]}(X, \mathbf{Z})$, permet de retrouver tous les champs de Picard (strictement commutatifs), définis par P. Deligne et A. Grothendieck dans [6]. Le champ $\operatorname{ch}(K)$ est constructible si et

(1) Justifions rapidement ces notations. Pour toute catégorie $\mathcal{C}$, on peut définir — fonctoriellement - un ensemble simplicial, son nerf, noté $\operatorname{Nerf}(\mathcal{C})$, dont la définition est rappelée par exemple dans [18, VI.2.1]. Si $\mathcal{C}$ est un groupoïde et $x$ un objet de $\mathcal{C}$, les $\pi_{i}$ de son nerf (pointé en $x$ ) sont nuls pour $i \geq 2$ (loc. cit. 2.6.2). Considérant les $\mathscr{F}(U)$ pour $U$ variable, on obtient, par faisceautisation, les ensembles définis plus haut. 
seulement si les faisceaux de cohomologie de $K$ le sont. Le $\pi_{0}$ correspond au $\underline{\mathrm{H}}^{0}$, et le $\pi_{1}$, pointé en la section nulle, au $\underline{H}^{-1}$. On a

$$
g^{*} \operatorname{ch}(K)=\operatorname{ch}\left(g^{*} K\right) \quad \text { et } \quad f_{*} \operatorname{ch}(K)=\operatorname{ch}\left(\tau_{\leq 0} \mathrm{R} f_{*} K\right) .
$$

Champs en catégories discrètes. — Soit $\mathscr{F}$ un faisceau d'ensembles sur X. On peut définir un champ $\mathrm{CD}(\mathscr{F})$ en prenant pour $\mathrm{CD}(\mathscr{F})(U)$ la catégorie discrète construite sur $\mathscr{F}(U)$. Ces groupoïdes sont «totalement disconnexes » en un sens évident. Dans le cas où $\mathscr{F}$ est un faisceau abélien, on a

$$
\operatorname{ch}(\mathscr{F}[0])=\operatorname{CD}(\mathscr{F}) .
$$

Le champ $\operatorname{CD}(\mathscr{F})$ est constructible si et seulement si $\mathscr{F}=\pi_{0}(\mathrm{CD}(\mathscr{F}))$ l'est (car les $\pi_{1}$ sont tous nuls). On a

$$
g^{*} \mathrm{CD}(\mathscr{F})=\operatorname{CD}\left(g^{*} \mathscr{F}\right) \quad \text { et } \quad f_{*} \operatorname{CD}(\mathscr{F})=\operatorname{CD}\left(f_{*} \mathscr{F}\right) \text {. }
$$

La situation est donc particulièrement simple. À partir de maintenant, nous considérerons tout faisceau $\mathscr{F}$ comme un champ en l'identifiant à $\mathrm{CD}(\mathscr{F})$.

Champs classifiant. - Soit $G$ un faisceau en groupes sur $X$. Rappelons qu'un $G$-torseur sur $X$ est un $X$-faisceau $T$ muni d'une action de $G$, qui est localement isomorphe ( $\operatorname{sur} X)$ à $G$ muni de l'action par translation sur lui-même. On en fait une catégorie en appelant «morphismes » les morphismes de $G$-faisceaux. Nous noterons Tors $(G)$ le champ dont la fibre en $U$ est la catégorie des $G_{\mid U}$-torseurs sur $U$. Dans le cas où $G$ est abélien, on a

$$
\operatorname{Tors}(G)=\operatorname{ch}(G[1]) \text {. }
$$

La terminologie «champs de Picard » se justifie par le fait qu'elle englobe le cas $G=\mathbf{G}_{m}$. Le champ Tors $(G)$, pour $G$ à nouveau quelconque, est constructible si et seulement si $G$ est constructible; en effet, $\pi_{1}$ (Tors $\left.(G)\right)$, pointé en le $G$-torseur trivial, n'est autre que $G$ et $\pi_{0}(\operatorname{Tors}(G))=\{\varnothing\}$. De même, si $G$ est un groupe constant fini de L-torsion, le champ Tors $(G)$ est ind-L-fini. On a

$$
g^{*} \operatorname{Tors}(G)=\operatorname{Tors}\left(G_{\mid Z}\right),
$$

mais $f_{*} \operatorname{Tors}(G)$ n'est pas nécessairement un champ du même type ${ }^{(2)}$ : cela dépend des propriétés géométriques du morphisme $f$. On montre aisément que

- $\pi_{0}\left(f_{*} \operatorname{Tors}(G)\right)=\mathrm{R}^{1} f_{*} G$ (cohomologie non abélienne) et

- $\pi_{1}\left(f_{*} \operatorname{Tors}(G)\right)=f_{*} G$ (pointé par l'image du revêtement trivial).

Soient $S$ un schéma, $f: X \rightarrow Y$ un $S$-morphisme entre $S$-schémas et $\mathscr{F}$ un champ sur $X$. Nous dirons que le couple $(f, \mathscr{F})$ est cohomologiquement propre relativement à $S$ si pour tout $S$-schéma $S^{\prime}$, la formation du champ $f_{*} \mathscr{F}$ commute au changement de base par le morphisme $Y^{\prime} \stackrel{\text { déf }}{=} Y \times{ }_{S} S^{\prime} \rightarrow Y$.

(2) Dans le cas où $G$ est abélien, cela résulte du fait que $\tau_{\leq 0}\left(\mathrm{R} f_{*} G[1]\right)$ n'est concentré en degré -1 que si $\mathrm{R}^{1} f_{*} G=0$, ce qui est faux en général.

TOME $131-2003-\mathrm{N}^{\mathrm{O}} 1$ 
ThÉORÈme 2.2. - Soient $S$ un schéma noethérien et $f: X \rightarrow Y$ un $S$ morphisme entre $S$-schémas de type fini. Alors :

a) Il existe un ouvert dense $U \hookrightarrow S$ vérifiant les conditions suivantes :

(i) pour tout ensemble fini $E$, le champ $f_{U *} E_{X_{U}}$ est constructible et le couple $\left(f_{U}, E_{X_{U}}\right)$ est cohomologiquement propre relativement à $U$;

(ii) pour tout $\mathrm{L}_{U}$-groupe fini $G$, le champ $f_{U *} \operatorname{Tors}\left(G_{X_{U}}\right)$ est constructible et le couple $\left(f_{U}\right.$, Tors $\left.\left(G_{X_{U}}\right)\right)$ est cohomologiquement propre relativement à $U$.

b) Pour tout champ $\mathscr{F}$ sur $X$, constructible, ind- $\mathrm{L}_{S}$-fini, il existe un ouvert dense $U \hookrightarrow S$ tel que le champ $f_{U * \mathscr{F}_{X_{U}}}$ soit constructible et que le couple $\left(f_{U}, \mathscr{F}_{X_{U}}\right)$ soit cohomologiquement propre relativement à $U$.

Remarque 2.3. - Si $f: X \rightarrow S$ est un morphisme de type fini, on peut montrer que l'image directe d'un faisceau d'ensembles constructible est constructible dès que $S$ est noethérien ( $c f$. [12]). Par contre, la constructibilité de $\mathrm{R}^{1} f_{*} \mathbf{Z} / n \mathbf{Z}$, avec $n$ inversible sur $S$, peut être mise en défaut avec $S$ noethérien (cf. loc. cit.); on conjecture que ce phénomène disparaît si $S$ est excellent.

En particulier,

Corollaire 2.4. - Soient $k$ un corps de caractéristique p et $f: X \rightarrow S=$ $\operatorname{Spec}(k)$ un $k$-schéma de type fini. Pour tout champ $\mathscr{F}$ sur $X$, constructible, ind-p $p^{\prime}$-fini, le champ $f_{*} \mathscr{F}$ est constructible et de formation compatible aux changements de base $Y \rightarrow S$.

Pour démontrer le théorème, nous ferons appel à un lemme de descente, dont l'énoncé nécessite quelques rappels. Un schéma simplicial $X$. est un foncteur contravariant de la catégorie $\Delta$, constituée des ensembles finis $[n]=\{0, \ldots, n\}$ où $n \geq 0$, munis des applications croissantes, dans la catégorie des schémas (cf. e.g. $[9$, ch. 1] dont nous reprenons les notations). À un tel objet est associé un topos étale noté $X_{\bullet}^{\sim}$ : ses objets sont les familles de faisceaux étales $F_{n}$ sur $X_{n}$, pour chaque $n \geq 0$, munies, pour chaque morphisme $\alpha:[n] \rightarrow[m]$, d'un $X_{\alpha}$-morphisme $F_{n} \rightarrow F_{m}$ (c'est-à-dire un morphisme $X_{\alpha}^{*} F_{n} \rightarrow F_{m}$ ) satisfaisants les conditions de transitivité évidentes pour les composés. Les champs sur ce topos admettent une description explicite : un champ $\mathscr{F}$ sur $X_{\bullet}^{\sim}$ correspond à la donnée d'un champ $\mathscr{F}_{n}$ sur chaque $X_{n}$, de 1-flèches $X_{\alpha}^{*} \mathscr{F}_{n} \rightarrow \mathscr{F}_{m}$ pour chaque morphisme $\alpha:[n] \rightarrow[m]$ dans $\Delta$ et d'isomorphismes de transitivité pour les composés, satisfaisant des conditions de cocycles pour les triplets d'applications composables.

Soit $\varepsilon: X . \rightarrow X$ un schéma simplicial augmenté vers $X$; on en déduit un morphisme entre les topos associés $: X_{\bullet}^{\sim} \rightarrow X^{\sim}$. Soit $\mathscr{F} \bullet$ un champ sur $X_{\bullet}$; le champ $\varepsilon_{*} \mathscr{F}$. se décrit comme la catégorie fibrée dont la fibre au-dessus d'un ouvert étale $U \rightarrow X$ est la catégorie des données de descente, c'est-à-dire 
des couples $(x, \varphi)$, où $x$ est un objet de $\mathscr{F}_{0}\left(U \times_{X} X_{0}\right)$ et $\varphi$ un isomorphisme $\left(\right.$ dans $\left.\mathscr{F}_{1}\left(U \times_{X} X_{1}\right)\right)$,

$$
\varphi: \partial_{0}^{*} x \stackrel{\sim}{\longrightarrow} \partial_{1}^{*} x
$$

satisfaisant la condition de cocycle sur $X_{2}\left(\right.$ dans $\left.\mathscr{F}_{2}\left(U \times_{X} X_{2}\right)\right)$ :

$$
\partial_{2}^{*} \varphi \circ \partial_{0}^{*} \varphi=\partial_{1}^{*} \varphi \text {. }
$$

Les $\partial_{i}$ désignent les morphismes déduits des applications strictement croissantes $d_{0}, d_{1}:\{0\} \rightarrow\{0,1\}$ et $d_{0}, d_{1}, d_{2}:\{0,1\} \rightarrow\{0,1,2\}$.

Pour la définition d'un hyper-recouvrement propre d'un schéma, nous renvoyons le lecteur à [7] ou [25].

Proposition 2.5 (O. Gabber). — Soit $\varepsilon: X_{\bullet} \rightarrow X$ un hyper-recouvrement propre d'un schéma $X$. Pour tout champ $\mathscr{F}$ sur $X^{\sim}$ le 1-morphisme d'adjonction $\alpha: \mathscr{F} \rightarrow \varepsilon_{*} \varepsilon^{*} \mathscr{F}$ est une équivalence.

C'est l'analogue non abélien du théorème de descente cohomologique propre de [25]. La flèche d'adjonction associe à tout objet $x$ sur $U / X$, son image inverse sur $X_{0}$, muni de la donnée de descente canonique.

Démonstration. - Commençons par la pleine fidélité des foncteurs $\alpha(U)$ pour tout ouvert étale $U \rightarrow X$. Comme $X{ }_{\bullet} \times{ }_{X} U$ est encore un hyper-recouvrement propre de $U$, on peut supposer $X=U$. De plus, par commutation de la formation des faisceaux de morphismes aux images directes et inverses ( $c f .[13$, II.3.2.8 et 3.1.5.3]), il suffit de démontrer la proposition pour un champ en catégories discrètes. Ce dernier cas résulte du théorème de descente [17, VIII. 9.1] qui traite le cas d'un cosquelette- 0 et du fait que le morphisme de topos induit par la surjection $\left(\partial_{0}, \partial_{1}\right): X_{1} \rightarrow X_{0} \times_{X} X_{0}$ est conservatif, si bien que les isomorphismes de faisceaux sur $X_{0} \times_{X} X_{0}$ se testent après changement de base à $X_{1}$.

Pour montrer que $\alpha$ est une équivalence, il suffit de le vérifier sur les fibres ( $c f$. [13, III.2.1.5.8]) ; on peut donc supposer que $X$ est un schéma strictement local. Commençons par le cas où $X$ est le spectre d'un corps séparablement clos $k$. On peut supposer $k$ algébriquement clos.

Si $X$. est le cosquelette du morphisme $X_{0} \rightarrow X$, c'est-à-dire $X_{n}=X_{0}^{\times_{k}(n+1)}$ pour tout $n \geq 0$, l'existence d'une section $s$ au morphisme $X_{0} \rightarrow X$ permet de montrer que toute donnée de descente est effective. Plus précisément, si $x_{0} \in \mathrm{Ob} \mathscr{F}_{X_{0}}\left(X_{0}\right)$ est muni d'une donnée de descente $\varphi$, le couple $\left(x_{0}, \varphi\right)$ est isomorphe à l'image inverse de $s^{*} x_{0} \in \mathrm{Ob} \mathscr{F}(X)$ sur $X_{0}$, muni de la donnée de descente canonique ( $f f$. e.g. $[5,6.1 .3])$. L'essentielle surjectivité du morphisme d'adjonction en découle.

Si l'on ne suppose plus que $X_{\bullet}=\operatorname{cosq}_{0}\left(X_{0} \rightarrow X\right)$, on dispose seulement, par adjonction, d'un morphisme $X_{\bullet} \rightarrow \operatorname{cosq}_{0}\left(X_{0} \rightarrow X\right)$; d'après ce qui précède,

TOME $131-2003-\mathrm{N}^{\mathrm{O}} 1$ 
il suffit de montrer que toute donnée de descente pour $X_{\text {. provient d'une donnée }}$ de descente sur $\operatorname{cosq}_{0}\left(X_{0} \rightarrow X\right)$. Soit $x_{0} \in \mathrm{Ob} \mathscr{F}_{X_{0}}\left(X_{0}\right)$ et

$$
\varphi: \partial_{0}^{*} x_{0} \stackrel{\sim}{\longrightarrow} \partial_{1}^{*} x_{0}
$$

un isomorphisme dans $\mathscr{F}_{X_{1}}\left(X_{1}\right)$, dont les images inverses satisfont la condition de cocycle dans $\mathscr{F}_{X_{2}}\left(X_{2}\right)$. Montrons que $\varphi$ se descend en un morphisme $\varphi^{\prime}$ sur $X_{0} \times_{X} X_{0}=X_{1}^{\prime}$; comme $X_{2} \rightarrow \operatorname{cosq}_{0}\left(X_{0} \rightarrow X\right)_{2}$ est surjectif, la condition de cocycle pour $\varphi^{\prime}$ résultera de celle pour $\varphi$, comme expliqué plus haut. D'après [17, VIII.9.2], appliqué au faisceau $\underline{\operatorname{Isom}}_{X_{1}^{\prime}}\left(\partial_{0}^{\prime *} x_{0}, \partial_{1}^{\prime *} x_{0}\right)$, il suffit, pour prouver l'existence de $\varphi^{\prime}$, de montrer que les deux images inverses de $\varphi$ sur $X_{1} \times_{X_{1}^{\prime}} X_{1}$ cö̈ncident. Notons $\partial_{1}^{\prime}, \partial_{2}^{\prime}: X_{1} \times_{X_{1}^{\prime}} X_{1} \rightrightarrows X_{1}$ les deux projections. Par hypothèse,

$$
\partial_{2}^{*} \varphi \circ \partial_{0}^{*} \varphi=\partial_{1}^{*} \varphi
$$

sur $X_{2}$; il s'agit de montrer que $\partial_{1}^{\prime *} \varphi=\partial_{2}^{\prime *} \varphi$. Posons $Z=X_{2} \times_{\partial_{0}, X_{1}, s_{0}} X_{0}$, et notons $i$ (resp. $p$ ) la projection $i: Z \rightarrow X_{2}$ (resp. $p: Z \rightarrow X_{0}$ ). Appliquons $i^{*}$ à la condition de cocycle; elle se réécrit

$$
i^{*} \partial_{1}^{*} \varphi=i^{*} \partial_{2}^{*} \varphi \circ p^{*} s_{0}^{*} \varphi .
$$

Comme $s_{0}^{*} \varphi=\operatorname{Id} \in \operatorname{Aut}_{X_{0}}\left(x_{0}\right)$, on a égalité des deux images inverses sur $Z$. Il nous suffit donc de montrer que le morphisme évident, défini par $\left(\partial_{1}, \partial_{2}\right)$, $Z \rightarrow X_{1} \times_{X_{1}^{\prime}} X_{1}$ est une surjection. Considérons les carrés cartésiens suivants :

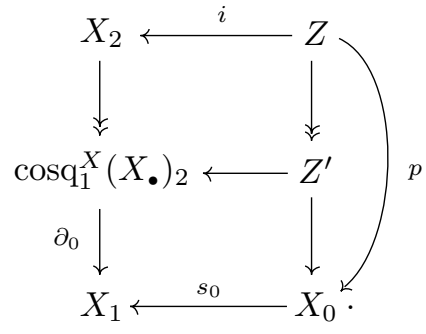

Le morphisme $Z \rightarrow Z^{\prime}$ est surjectif; il reste à montrer qu'il s'identifie au morphisme $\left(\partial_{1}, \partial_{2}\right): Z \rightarrow X_{1} \times_{X_{1}^{\prime}} X_{1}$. Rappelons (cf. [9, p. 10]) que

$$
\operatorname{cosq}_{1}^{X}\left(X_{\bullet}\right)_{2}=\left(X_{1} \times_{X_{0}} X_{1}\right) \times_{X_{1}^{\prime}} X_{1},
$$

de sorte que le morphisme $\partial_{0}: \operatorname{cosq}_{1}^{X}\left(X_{\bullet}\right)_{2} \rightarrow X_{1}$ correspond à la première projection $X_{1} \times_{X_{0}} X_{1} \rightarrow X_{1}$. On en déduit immédiatement le résultat.

BULLETIN DE LA SOCIÉtÉ MATHÉMATIQUE DE FRANCE 
Si maintenant $X$ est strictement local de point fermé $x$, on a un diagramme commutatif de foncteurs

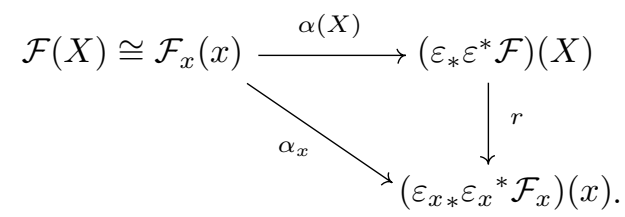

On vient de prouver que $\alpha_{x}$ est une équivalence; d'après le théorème de changement de base propre pour les faisceaux d'ensembles, appliqué aux faisceaux d'homomorphismes, le foncteur $r$ est pleinement fidèle. Ainsi, $\alpha$ est une équivalence.

LEMme 2.6. - Soient $S$ un schéma noethérien et $f: X \rightarrow Y$ un $S$-morphisme séparé entre $S$-schémas de type fini, avec $Y$ séparé. Il existe un ouvert dense $U \hookrightarrow S$ et un homéomorphisme universel $U^{\prime} \rightarrow U$ tels qu'après changement de base par $U^{\prime} \rightarrow S$, on ait un diagramme

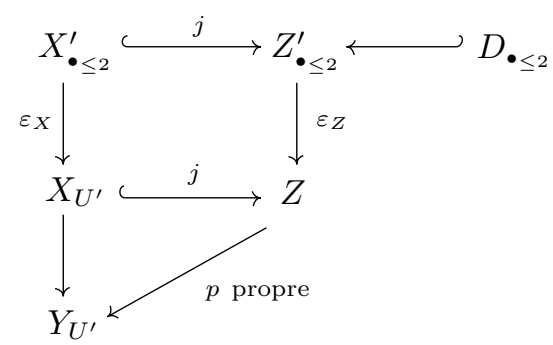

où $j$ est une immersion ouverte dominante, $\varepsilon_{Z}$ un hyper-recouvrement propre tronqué à l'ordre 2 , et $D_{\bullet \leq 2}$ un diviseur à croisements normaux relativement à $U^{\prime}$ dans le schéma simplicial tronqué $Z_{\bullet \leq 2}^{\prime}$ lisse sur $U^{\prime}$, de complémentaire $X_{\bullet \leq 2}^{\prime}$.

Démonstration. - Cas $S=\operatorname{Spec}(k)$, avec $k$ parfait. Soit $X \stackrel{j}{\rightarrow} Z \stackrel{p}{\rightarrow} Y$ une factorisation de $f$ en une immersion ouverte dominante et un morphisme propre.

Montrons qu'il existe un morphisme propre et surjectif $a: Z_{0} \rightarrow Z$, avec $Z_{0}$ régulier (donc lisse sur $k$ ) tel que l'image inverse de $X$ dans $Z_{0}$ soit le complémentaire d'un diviseur à croisements normaux. Le théorème [21, 3.1] affirme que si $Z$ est intègre séparé, il existe un morphisme $r: Z_{0} \rightarrow Z$ propre, surjectif (génériquement étale) et une compactification (projective) $\bar{Z}_{0}$ de $Z_{0}$ telle que $\left(\bar{Z}_{0}-Z_{0}\right) \cup r^{-1}(Z-X)$ soit un diviseur à croisements normaux stricts dans $\bar{Z}_{0}$; en particulier, $r^{-1}(X)$ est le complémentaire d'un diviseur à croisements normaux dans $Z_{0}$. Voyons comment cela implique notre résultat. Soit

$$
b: Z^{\prime} \stackrel{\text { déf }}{=} \coprod\left(Z_{\alpha}\right)_{\text {réd }} \longrightarrow Z
$$

TOME $131-2003-\mathrm{N}^{\mathrm{O}} 1$ 
le morphisme canonique, où les $Z_{\alpha}$ sont les composantes irréductibles (en nombre fini) de $Z$. Le morphisme $b$ est fini et surjectif. Les $\left(Z_{\alpha}\right)_{\text {réd }}$ sont intègres donc il existe une altération $c_{\alpha}: Z_{\alpha 0} \rightarrow\left(Z_{\alpha}\right)_{\text {réd }}$ comme précédemment, associée à l'ouvert $\left(Z_{\alpha}\right)_{\text {réd }} \times_{Z} X$. Le coproduit des $c_{\alpha}$ définit un morphisme

$$
c: Z_{0}^{\prime} \stackrel{\text { déf }}{=} \coprod Z_{\alpha 0} \rightarrow Z^{\prime}
$$

propre et surjectif; le morphisme $a=b c: Z_{0}^{\prime} \rightarrow Z$ satisfait les conditions précédentes.

Finalement, utilisant les techniques de [7], on construit de même $Z_{1}^{\prime}$ et $Z_{2}^{\prime}$. Ceci achève la démonstration du lemme dans ce cas.

Cas général. - On peut supposer $S$ connexe, puis irréductible et enfin intègre quitte à faire un changement de base par un homéomorphisme universel. Soit $\eta_{S}$ le point générique de $S$, et $\bar{\eta}_{S}$ le spectre d'une clôture parfaite de $\kappa\left(\eta_{S}\right)$. D'après le cas précédent, il existe une compactification de $f_{\bar{\eta}_{S}}$ ainsi que des hyper-recouvrements tronqués comme dans l'énoncé, sur $\bar{\eta}_{S}$. Comme il s'agit d'hyper-recouvrements tronqués, on peut descendre ces constructions à une extension finie de $\eta_{S}$, puis les étendre à un $S$-schéma dominant, génériquement fini et radiciel $U^{\prime}$, que l'on peut supposer s'envoyant sur son image par un homéomorphisme universel.

On peut démontrer le même résultat sans hypothèse de séparation sur $Y$. Il est aussi possible d'étendre l'hyper-recouvrement tronqué de $X$ en un hyperrecouvrement propre, mais les images directes ne dépendent que de ces tronqués à l'ordre 2 .

Revenons maintenant à la démonstration du théorème 2.2. Ici, comme souvent par la suite, on notera des équivalences avec des égalités.

Il est démontré dans [24, p. 59-65], comment déduire le b) du a). Nous allons donc démontrer a), en supposant le morphisme $f$ et le schéma $Y$ séparés. Soient $U$ et $U^{\prime}$ comme dans 2.6 - dont nous reprenons les notations - ; vérifions que a) vaut sur $U$. Comme le morphisme $U^{\prime} \rightarrow U$ est un homéomorphisme universel, il suffit de démontrer le résultat sur $U^{\prime}$. On peut donc supposer $S=U=U^{\prime}$. On dispose alors d'hyper-recouvrements (tronqués)

$$
\varepsilon_{X}: X_{\bullet \leq 2} \rightarrow X \quad \text { et } \quad \varepsilon_{Z}: Z_{\bullet \leq 2} \rightarrow Z
$$

des schémas $X$ et $Z$, ainsi que des schémas qui s'en déduisent par changement de base relativement à $S$. Soit maintenant $S^{\prime} \rightarrow S$ un morphisme, et $\mathscr{F}$ comme dans l'énoncé. On note $g_{Y}$ (resp. $g_{X}$, resp. $g_{X}$ ) la flèche de changement de base $Y_{S^{\prime}} \rightarrow Y$ (resp. $X_{S^{\prime}} \rightarrow X$, resp. $X_{\bullet \leq 2} \times_{S} S^{\prime} \rightarrow X_{\bullet \leq 2}$ ). Calculons $g_{Y}^{*} f_{*} \mathscr{F}$; on a, d'après 2.5 et par commutativité des diagrammes,

$$
g_{Y}^{*} f_{*} \mathscr{F}=g_{Y}^{*} f_{*} \varepsilon_{X *} \varepsilon_{X}^{*} \mathscr{F}=g_{Y}^{*} p_{*} \varepsilon_{Z_{*}} j_{*}\left(\varepsilon_{X}^{*} \mathscr{F}\right) .
$$

BULletin DE LA SOCIÉtÉ MATHÉMATIQUE DE FRANCE 
Rappelons que l'on a un théorème de changement de base propre pour les champs ind-finis ([13, VII.2.2.2]). Ainsi, utilisant la propreté de $p$ et de l'hyperrecouvrement, on a

$$
g_{Y}^{*} f_{*} \mathscr{F}=p_{*}^{\prime} \varepsilon_{Z^{\prime} *} g_{X \bullet}^{*} j_{*}\left(\varepsilon_{X}^{*} \mathscr{F}\right) .
$$

Le théorème de propreté cohomologique $[24,2.4]^{(3)}$ (joint à la propreté de $\varepsilon_{X}$ sur $X$ ), affirme en particulier que

$$
g_{X_{\bullet}}^{*} j_{*}\left(\varepsilon_{X}^{*} \mathscr{F}\right)=j^{\prime *} \varepsilon_{X^{\prime}}^{*}\left(g_{X}^{*} \mathscr{F}\right) .
$$

On peut conclure en utilisant à nouveau le résultat de descente, sur $X_{S^{\prime}}$. Pour traiter le cas général, on se ramène au cas où $Y$ est affine (la question est locale sur $Y$ ), donc séparé. Considérant un recouvrement ouvert affine de $X$ et l'hyper-recouvrement associé, on se ramène aussitôt au cas où $X$ est affine, donc $f$ séparé.

Il nous faut maintenant démontrer la constructibilité. Commençons par rappeler le lemme :

Lemme 2.7 (voir $[24,3.1 .1])$ - — Soient $\varepsilon: X_{\bullet} \rightarrow X$ un schéma simplicial augmenté et $\mathscr{F}$ un champ sur $X_{\bullet}$. Pour que le champ $\varepsilon_{*} \mathscr{F}$ soit constructible, il suffit que $\varepsilon_{0 *} \mathscr{F}_{0}$ et $\varepsilon_{1 *} \mathscr{F}_{1}$ le soient.

En effet, dans le langage de loc. cit., on a un diagramme exact de champs :

$$
\varepsilon_{*} \mathscr{F} \longrightarrow\left[\varepsilon_{0 *} \mathscr{F}_{0} \rightrightarrows \varepsilon_{1 *} \mathscr{F}_{1}\right] .
$$

En utilisant cette fois-ci le théorème de constructibilité des images directes par un morphisme propre (loc. cit., 6.2) ainsi que la constructibilité dans le cas d'une l'immersion ouverte de complémentaire un diviseur à croisements normaux (loc. cit., 2.4 2 ), l'énoncé de constructibilité du a) se démontre comme précédemment.

REMARQUe 2.8. - Dans la première version de ce texte, on donnait une autre démonstration, par « approximations successives », du théorème 2.2, indépendante de la proposition 2.5. Pour cela, on commence par montrer que le morphisme de changement de base est fidèle, du moins génériquement sur la base $S$. Il suffit de considérer un hyper-recouvrement tronqué à l'ordre 0 convenable (i.e. comme dans 2.6) $X_{0} \rightarrow X$, et d'utiliser les deux théorèmes de propreté cohomologique rappelés plus haut. Fort de ce résultat générique, que l'on applique ensuite aux constituants du 0 -cosquelette tronqué à l'ordre $1, \operatorname{cosq}_{0}\left(X_{0} \rightarrow X\right)_{\bullet<1}$, on démontre la pleine fidélité générique des flèches de changement de base et enfin le fait que ce sont des équivalences, en utilisant cette fois le tronqué à l'ordre 2. De même pour la constructibilité.

(3) L'hypothèse de modération, dans le cas d'un champ de torseurs, est automatique ici puisque le groupe structural est supposé d'ordre premier aux caractéristiques résiduelles. 


\section{Locale acyclicité générique}

ThÉorème 3.1 (O. Gabber). — Soient $S$ un schéma noethérien et $f: X \rightarrow S$ un morphisme de type fini. Il existe un ouvert dense $U \hookrightarrow S$ tel que pour tout morphisme cohérent ${ }^{(4)} \mathrm{g}: Y \rightarrow Z$ de U-schémas, et tout champ $\mathscr{F}$ ind- $\mathrm{L}_{Z}$ fini sur $Y$, le morphisme de changement de base $p^{*} g_{*} \mathscr{F} \rightarrow g^{\prime}{ }_{*} p^{*} \mathscr{F}$ soit une équivalence, où $g^{\prime}, p$ et $p^{\prime}$ sont les morphismes définis par le carré cartésien ci-dessous :

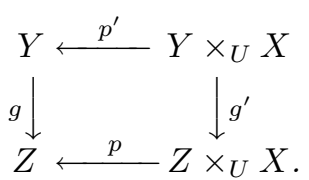

Démonstration. - Soient $U$ et $U^{\prime}$ comme dans 2.6 - dont nous reprenons les notations - ; montrons que la conclusion du théorème vaut sur $U$. Comme expliqué plus haut, on peut supposer $U^{\prime}=U$, et finalement, pour alléger les notations, $S=U$. Soient $g$ et $\mathscr{F}$ comme dans l'énoncé ; considérons le diagramme cartésien suivant :

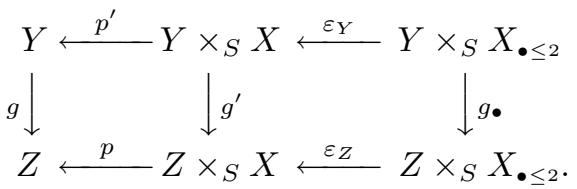

D'après le théorème de changement de base lisse pour les champs (voir [13, 2.1.2]), la formation de l'image directe d'un champ (ind-fini, de torsion première aux caractéristiques résiduelles) par un morphisme cohérent commute aux changements de bases par des morphismes lisses. Le foncteur de changement de base $\left(p \varepsilon_{Z}\right)^{*} g_{*} \mathscr{F} \rightarrow g_{\bullet *}\left(p^{\prime} \varepsilon_{Y}\right)^{*} \mathscr{F}$ est donc une équivalence. Le morphisme de champs $p^{*} g_{*} \mathscr{F} \rightarrow g^{\prime}{ }_{*} p^{\prime *} \mathscr{F}$ s'identifie, par 2.5, à l'image directe du précédent par $\varepsilon_{Z_{*}}$; c'est donc une équivalence.

Pour tout schéma $X$ et tout point géométrique $x$ de $X$, on note $X_{(x)}$ le localisé strict de $X$ en $x$.

DÉFInItion 3.2. - Soit $f: X \rightarrow S$ un morphisme et $\mathscr{F}$ un champ sur $X$. On dit que $(f, \mathscr{F})$ est localement 1 -acyclique (resp. 0-acyclique, resp. -1-acyclique) si pour tout point géométrique $x$ de $X$ d'image $s$ par $f$, et toute générisation $t$ de $s$ (i.e. un point géométrique de $\left.S_{(s)}\right)$, le foncteur $\mathscr{F}\left(X_{(x)}\right) \rightarrow \mathscr{F}\left(X_{(x) t}\right)$ est une équivalence (resp. pleinement fidèle, resp. fidèle).

Les schémas $X_{(x) t}$ seront appelés fibres de Milnor du morphisme $X \rightarrow S$. On dira souvent localement acyclique au lieu de localement 1-acyclique.

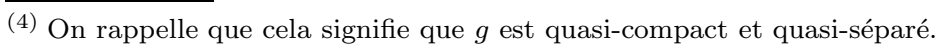

BULLETIN DE LA SOCIÉTÉ MATHÉMATIQUE DE FRANCE 
ThÉORÈme 3.3. - Soient $S$ un schéma noethérien et $f: X \rightarrow S$ un morphisme de type fini. Alors,

a) Il existe un ouvert dense $U \hookrightarrow S$ vérifiant les conditions suivantes :

(i) pour tout ensemble fini $E$, le couple $\left(f, E_{X}\right)$ est universellement localement acyclique;

(ii) pour tout $\mathrm{L}_{S}$-groupe fini $G$, le couple $\left(f\right.$, Tors $\left.\left(G_{X}\right)\right)$ est universellement localement acyclique.

b) Pour tout champ $\mathscr{F}$ sur $X$ constructible, ind- $\mathrm{L}_{S}$-fini, il existe un ouvert dense $U \hookrightarrow S$ tel que le couple $\left(f_{U}, \mathscr{F}_{X_{U}}\right)$ soit universellement localement acyclique.

Démonstration. - L'universalité résultera de la démonstration. Commençons par démontrer a). Soit $U$ un ouvert dense de $S$ pour lequel les conclusions du théorème précédent sont valables (relativement au morphisme $f: X \rightarrow S$ ) et montrons que l'on a (i) et (ii). Soient $E$ un ensemble fini (resp. $G$ un $\mathrm{L}_{U}$-groupe fini), $x$ un point géométrique de $X_{U}$ et $t, s$ comme plus haut; considérons le carré cartésien

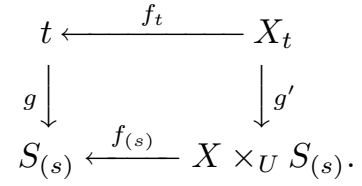

Notons $\mathscr{F}$ le champ $E_{t}$ (resp. Tors $\left.\left(G_{t}\right)\right)$ sur $t$. Le morphisme $f_{(s)}^{*} g_{*} \mathscr{F} \rightarrow g^{\prime} f_{t}^{*} \mathscr{F}$ est une équivalence; calculons sa fibre en $x$. Comme $x$ s'envoie sur $s$, on a

$$
\left(f_{(s)}^{*} g_{*} \mathscr{F}\right)_{x}=\left(g_{*} \mathscr{F}\right)_{s}=\mathscr{F}(t)
$$

tandis que $\left(g^{\prime}{ }_{*} f_{t}^{*} \mathscr{F}\right)_{x}=\mathscr{F}\left(X_{(x) t}\right)$ avec les notations de loc. cit. Cela démontre que $X_{(x) t}$ est connexe (resp. $\mathrm{L}_{U}$-simplement connexe) d'où le résultat.

Venons-en au cas général, i.e. b).

Commençons par le cas d'un champ en catégories discrètes $\mathscr{F}=E$. On sait qu'il existe un morphisme fini $\pi: X^{\prime} \rightarrow X$, un faisceau d'ensembles $F$ constructible, constant sur chaque composante connexe de $X^{\prime}$ - nous dirons presque constant - et un monomorphisme

$$
E \longrightarrow \pi_{*} F \stackrel{\text { déf }}{=} E^{\prime} \text {. }
$$

Ainsi $E=\pi_{0}(\mathscr{F})$ est le noyau de la double flèche naturelle

$$
E^{\prime} \Longrightarrow E^{\prime} \coprod_{E} E^{\prime} \stackrel{\text { déf }}{=} E^{\prime \prime} \text {. }
$$

Plongeant cette fois $E^{\prime \prime}$ dans l'image directe d'un faisceau presque constant par un morphisme fini, on exprime $E$ comme noyau d'une double flèche entre faisceaux constructibles du type précédent; il suffit, par exactitude, de vérifier le théorème pour ces faisceaux.

TOME $131-2003-\mathrm{N}^{\mathrm{O}} 1$ 
Soient $\pi: X^{\prime} \rightarrow X$ un morphisme fini et $E$ l'image directe par $\pi$ d'un faisceau constructible $F$ presque constant. D'après a), il existe un ouvert dense $U$ de $S$ tel que les fibres de Milnor de $X_{U}^{\prime} \rightarrow U$ soient connexes. Montrons que $U$ convient pour $E$. Si $x$ est un point géométrique de $X_{U}^{\sim}$,

$$
E\left(X_{(x)}\right)=F\left(X^{\prime} \times_{X} X_{(x)}\right)=F\left(\coprod_{x^{\prime} \rightarrow x} X_{\left(x^{\prime}\right)}^{\prime}\right) .
$$

Il ne reste plus qu'à rappeler que les « restrictions » $F\left(X_{\left(x^{\prime}\right)}^{\prime}\right) \rightarrow F\left(X_{\left(x^{\prime}\right) t}^{\prime}\right)$ sont des isomorphismes.

Soit maintenant $\mathscr{F}$ un champ sur $X$ constructible, ind- $\mathrm{L}_{S}$-fini. Il existe un nombre fini d'ouverts étales $U_{i} \rightarrow X(i \in\{1, \ldots, r\}=I)$ et des sections $s_{i} \in \mathrm{Ob} \mathscr{F}\left(U_{i}\right)$ qui engendrent $\pi_{0}(\mathscr{F})$ (i.e. telles que pour tout point géométrique $x$ de $X$, et toute section $s_{x} \in \mathrm{Ob} \mathscr{F}_{x}$, il existe un indice $i$ tel que $x$ se factorise par $U_{i}$ et que $s_{x}$ soit isomorphe à $s_{i x}$ ). Pour tout $i, j \in I$, notons $U_{i, j}=U_{i} \times_{X} U_{j}$ et $F_{i, j}$ le $U_{i, j}$-faisceau $\underline{\operatorname{Isom}}\left(s_{i \mid U_{i, j}}, s_{j \mid U_{i, j}}\right)$. Soit $U$ un ouvert dense de $S$ tel que b) soit valable pour tous ces faisceaux; il en existe par ce qui précède. Soient $x$ un point géométrique de $X_{U}, t$ une générisation de $f_{(x)}$ et $\alpha, \beta$ deux objets de $\mathscr{F}\left(X_{(x)}\right)$. Montrons que le morphisme $\operatorname{Hom}_{X_{(x)}}(\alpha, \beta) \rightarrow$ $\operatorname{Hom}_{X_{(x) t}}\left(\alpha_{t}, \beta_{t}\right)$ est un isomorphisme; cela démontrera que le couple $\left(f_{U}, \mathscr{F}_{X_{U}}\right)$ est localement 0 -acyclique. Deux cas se présentent : soit il existe un indice $i$ tel que $\alpha \cong \beta \cong s_{i x}$, auquel cas la conclusion résulte de l'hypothèse faite sur $F_{i, i}=\operatorname{Aut}_{U_{i, i}}\left(s_{i}\right)$, soit $\alpha$ et $\beta$ ne sont pas isomorphes, et il faut montrer qu'ils ne le deviennent pas après restrictions à $X_{(x) t}$. Il existe $i, j$ tels que $\alpha \cong s_{i_{x}}$ et $\beta \cong s_{j_{x}}$; si l'on note encore $x$ un point géométrique de $U_{i, j}$ relevant $x$, on a

$$
\begin{gathered}
\varnothing=\operatorname{Hom}_{X_{(x)}}(\alpha, \beta)=F_{i, j}\left(U_{i, j(x)}\right) \quad \text { et } \\
\operatorname{Hom}_{X_{(x) t}}\left(\alpha_{t}, \beta_{t}\right)=F_{i, j}\left(U_{i, j(x) t}\right)=\varnothing, \quad \text { CQFD. }
\end{gathered}
$$

La locale 0-acyclicité (générique) étant acquise, nous pouvons supposer, d'après [13, III.2.1.5.9], que $\mathscr{F}$ est la gerbe des $\underline{\operatorname{Aut}}\left(s_{i}\right)$-torseurs sur $U_{i}$. Comme $I$ est fini, cela nous ramène à démontrer le b) dans le cas particulier d'un champ de torseurs sous un groupe constructible ind- $\mathrm{L}_{S}$-fini, noté $\mathscr{G}$. Il existe un monomorphisme de faisceaux en groupes $\mathscr{G} \hookrightarrow \mathscr{G}^{\prime}$, où $\mathscr{G}^{\prime}$ est l'image directe par un morphisme fini $\pi: X^{\prime} \rightarrow X$ d'un faisceau en groupe presque constant $\mathrm{L}_{S^{-}}$ fini $G$. Il résulte de la suite exacte longue tronquée de cohomologie non abélienne $[1,3.1]$, qu'il suffit de traiter le cas de Tors $\left(\mathscr{G}^{\prime}\right)$ et de $\mathscr{G}^{\prime} / \mathscr{G}$. Quitte à restreindre encore $S$, on peut supposer le résultat acquis pour $\mathscr{G}^{\prime} / \mathscr{G}$. D'après a) (ii), on peut aussi supposer que les fibres de Milnor du morphisme $X^{\prime} \rightarrow S$ sont $\mathrm{L}_{S^{-}}$ simplement connexe. Soient maintenant $x, s, t$ comme dans l'énoncé ; on veut montrer que $\mathrm{H}^{1}\left(X_{(x) t}, \pi_{*} G\right)=\{e\}$. Or

$$
\mathrm{H}^{1}\left(X_{(x) t}, \pi_{*} G\right)=\mathrm{H}^{1}\left(X^{\prime} \times_{X} X_{(x) t}, G\right)=\mathrm{H}^{1}\left(\coprod_{x^{\prime} \rightarrow x} X_{\left(x^{\prime}\right) t}^{\prime}, G\right)=\{e\},
$$

le premier isomorphisme vient du fait que le morphisme $\pi$ est fini.

BULLETIN DE LA SOCIÉTÉ MATHÉMATIQUE DE FRANCE 
Cette dernière réduction ( $\mathrm{du}$ cas d'un groupe constructible à un groupe constant) est due à Ofer Gabber. Dans la première version de ce texte, le a) était démontré sans utiliser le théorème 3.1. Remarquons par exemple que l'on peut déduire a) (i) des énoncés abéliens de P. Deligne en utilisant, si $\ell$ est un nombre premier inversible sur $S$, l'adjoint à gauche du foncteur d'oubli

$\{$ faisceaux abéliens de $\ell$-torsion $\} \longrightarrow X^{\sim}$,

parfois noté $E \mapsto \mathbf{F}_{\ell}[E]$.

REMARque 3.4 (variante abélienne de 3.1). - Soient $S$ un schéma noethérien, $X$ un schéma séparé et $f: X \rightarrow S$ un morphisme de type fini. Il existe un ouvert dense $U$ de $S$ tel que pour tout morphisme cohérent $g: Y \rightarrow Z$ de $U$-schémas, tout $n$ un entier inversible sur $S$, et tout faisceau constructible $\mathscr{F}$ de $\mathbf{Z} / n \mathbf{Z}$-modules sur $Y$, le morphisme $p^{*} \mathrm{R} g_{*} \mathscr{F} \rightarrow \mathrm{R} g^{\prime}{ }_{*} p^{*} \mathscr{F}$ soit un isomorphisme. A priori, on s'attendrait à un énoncé tronqué. Cependant, si $r$ est un entier supérieur à la dimension des fibres de $f$, la $r$-acyclicité locale générique, qui se démontre à l'aide d'un hyper-recouvrement tronqué, entraîne l'acyclicité locale sur le même ouvert ( $c f$. [3, 1.17], et le fait que $r$ majore la dimension cohomologique des fibres de Milnor qui apparaissent).

On peut aussi retrouver par la même méthode (i.e. descente cohomologique abélienne et altérations) les deux théorèmes génériques de [8].

\section{Locale constance générique et applications au $\pi_{1}^{p \prime}$}

LEMme 4.1. - Soient $X$ un schéma et $\mathscr{F}$ un champ sur $X$ constructible. Les conditions suivantes sont équivalentes:

(i) pour tout point géométrique $x$ de $X$ et toute générisation $y \rightarrow x$, le morphisme $\mathscr{F}\left(X_{(x)}\right) \rightarrow \mathscr{F}\left(X_{(y)}\right)$ est une équivalence;

(ii) le faisceau $\pi_{0}(\mathscr{F})$ et, pour toute section locale $s$, les faisceaux $\pi_{1}(\mathscr{F}, s)$ sont localement constants.

Démonstration. - Que la première condition entraîne la seconde résulte de $[2,2.11]$.

Pour l'autre implication, on commence par remarquer que l'essentielle surjectivité de ces foncteurs découle de la surjectivité de

$$
\pi_{0}(\mathscr{F})\left(X_{(x)}\right) \longrightarrow \pi_{0}(\mathscr{F})\left(X_{(y)}\right) .
$$

(Bien que $\pi_{0}(\mathscr{F})$ ne soit que le faisceau associé au préfaisceau des classes d'isomorphismes, cette difficulté disparaît car les schémas considérés sont strictement locaux.) Soit $y \rightarrow x$ une générisation et $s, s^{\prime} \in \mathrm{Ob} \mathscr{F}\left(X_{(x)}\right)$; montrons que

$$
\operatorname{Hom}_{x}\left(s, s^{\prime}\right)=\operatorname{Hom}_{y}\left(s_{y}, s_{y}^{\prime}\right) \text {. }
$$

Si $s \cong s^{\prime}$, on utilise l'hypothèse faite sur $\pi_{1}(\mathscr{F}, s)$ tandis que si $s$ et $s^{\prime}$ ne sont pas isomorphes, cela résulte de l'injectivité de $\pi_{0}(\mathscr{F})\left(X_{(x)}\right) \rightarrow \pi_{0}(\mathscr{F})\left(X_{(y)}\right)$.

TOME $131-2003-\mathrm{N}^{\mathrm{O}} 1$ 
Un champ satisfaisant les deux conditions précédentes est dit localement constant.

Suivant [19], on peut définir - dans certains cas — des flèches de spécialisation et de cospécialisation de la façon suivante. Si $(f, \mathscr{F})$ est cohomologiquement propre et $t \rightarrow s$ une spécialisation sur $S$ (comme dans 3.2), $\mathscr{F}\left(X \times_{S} S_{(s)}\langle t\rangle\right) \rightarrow \mathscr{F}\left(X_{s}\right)$ est une équivalence, où $S_{(s)}\langle t\rangle$ désigne le normalisé de $S_{(s)}$ dans $t$ (cf. loc. cit., 1.1). Un foncteur de spécialisation est défini en composant une équivalence quasi-inverse $\mathscr{F}\left(X_{s}\right) \rightarrow \mathscr{F}\left(X \times_{S} S_{(s)}\langle t\rangle\right)$ avec le foncteur $\mathscr{F}\left(X \times{ }_{S} S_{(s)}\langle t\rangle\right) \rightarrow \mathscr{F}\left(X_{t}\right)$. Si l'on suppose cette fois $(f, \mathscr{F})$ localement acyclique, on définit de manière analogue un foncteur de cospécialisation $\mathscr{F}\left(X_{t}\right) \rightarrow \mathscr{F}\left(X_{s}\right)$ pour toute spécialisation $t \rightarrow s$. Lorsque les flèches de spécialisation et cospécialisation sont définies, leurs composés sont 2-isomorphes à l'identité.

Finalement, on trouve (cf. loc. cit., 2) :

Proposition 4.2. - Soient $f: X \rightarrow S$ un morphisme cohérent et $\mathscr{F}$ un champ sur $X$ tel que $(f, \mathscr{F})$ soit cohomologiquement propre et localement acyclique. Alors, $f_{*} \mathscr{F}$ est localement constant.

Remarquons que si $\mathscr{F}$ est un champ en catégories discrètes, le couple $(f, \mathscr{F})$ sera localement acyclique si le faisceau $\mathscr{F}$ est localement constant et $(f, E)$ est localement acyclique pour un ensemble fini $E$ de cardinal supérieur à 2 .

Grâce aux deux théorèmes génériques, on en déduit :

Corollaire 4.3. - Soient $S$ un schéma noethérien, $f: X \rightarrow S$ un morphisme de type fini, et $\mathscr{F}$ un champ constructible, ind- $\mathrm{L}_{S}$-fini sur $X$. Il existe un ouvert dense $U$ de $S$ tel que $f_{U *} \mathscr{F}_{X_{U}}$ soit localement constant, constructible et de formation compatible à tout changement de base.

Corollaire 4.4. - Soient $S$ un schéma noethérien et $f: X \rightarrow S$ un morphisme séparé de type fini. Il existe un ouvert dense $U \hookrightarrow S$ tel que pour tout $\mathrm{L}_{S}$-groupe fini $G$, toute générisation $t \rightarrow s$ de points géométriques de $U$ les flèches

$$
\mathrm{H}^{1}\left(X_{s}, G\right) \longleftarrow\left(\mathrm{R}^{1} f_{*} G\right)_{s} \cong \mathrm{H}^{1}\left(X_{S_{(s)}}, G\right) \longrightarrow \mathrm{H}^{1}\left(X_{t}, G\right)
$$

soient des isomorphismes.

Cela résulte immédiatement de 4.3. La traduction de cet énoncé en termes plus géométriques est immédiate :

Si $S$ est un schéma noethérien intègre de point générique $\eta$ et $f: X \rightarrow S$ un morphisme de type fini de fibre générique géométrique connexe, il existe un ouvert dense $U \hookrightarrow S$ tel que pour chaque point géométrique $\bar{u}$ de $U$, le schéma $X_{\bar{u}}$ soit connexe et de groupe fondamental $\mathrm{L}_{S}$-primaire isomorphe $\grave{a} \pi_{1}^{\mathrm{L}_{S}}\left(X_{\bar{\eta}}\right)$. 
On laisse le soin au lecteur de préciser cet énoncé en choisissant des points bases et des chemins.

Corollaire 4.5. - Soient $k$ un corps algébriquement clos d'exposant caractéristique $p, X$ un $k$-schéma de type fini et $x$ un $k$-point de $X$. Pour toute extension $K / k$, avec $K$ algébriquement clos, le morphisme canonique $\pi_{1}^{p \prime}\left(X_{K}, x\right) \rightarrow$ $\pi_{1}^{p \prime}\left(X_{k}, x\right)$ est un isomorphisme.

Démonstration. - Cela résulte immédiatement du fait que si l'on note $f$ le morphisme $X \rightarrow \operatorname{Spec}(k)$, pour tout $p^{\prime}$-groupe fini $G$, la formation du faisceau $\mathrm{R}^{1} f_{*} G$ commute à tous les changements de bases $S \rightarrow \operatorname{Spec}(k)(c f .2 .4)$ et en particulier à $\operatorname{Spec}(K) \rightarrow \operatorname{Spec}(k)$.

Soient $X$ un schéma noethérien et $\mathrm{L}$ un ensemble de nombres premiers. Suivant Artin-Mazur, on peut définir un pro-objet, noté $X_{\text {ét }}^{\mathrm{L}}$, de la catégorie homotopique des CW-complexes. Pour la définition de ce pro-objet, nous renvoyons à $[4,3.4$ et 4.2$]$ pour la définition des Ł-équivalences.

Corollaire 4.6. - Soient $S$ un schéma noethérien et $f: X \rightarrow S$ un morphisme de type fini. Il existe un ouvert dense $U \hookrightarrow S$ tel que pour toute flèche de spécialisation $t \rightarrow s$ de points géométriques de $U$, les morphismes

$$
X_{s} \longrightarrow X \times_{S} S_{(s)}\langle t\rangle \longleftarrow X_{t}
$$

induisent des দ-équivalences $X_{s e ́ t}^{\mathrm{L}_{U}} \cong X_{t e ́ t}^{\mathrm{L}_{U}}$.

Suivant Sullivan, on peut aussi définir fonctoriellement, pour chaque schéma $X$, un vrai type d'homotopie holim $X_{\text {ét }}^{\mathrm{L}}$; après application de ce foncteur, les morphismes précédents deviennent des équivalences d'homotopie.

Démonstration. - Compte-tenu des propositions 5.8, 5.9 et 6.4 de [9], qui permettent de comparer faisceaux lisses sur $X^{\sim}$ et sur $X_{\text {ét }}^{\mathrm{L}}$ ainsi que la cohomologie de ces topos à valeurs dans ces faisceaux, le théorème d'Artin-Mazur-Quillen (loc. cit., 6.5) prend la forme qui suit :

Si $g: Y \rightarrow Z$ est un morphisme de schémas noethériens, et $\mathrm{L}$ un ensemble de nombres premiers, $g^{\mathrm{L}}: Y_{\text {ét }}^{\mathrm{L}} \rightarrow Z_{\text {ét }}^{\mathrm{L}}$ est une q-équivalence si et seulement si :

(i) le morphisme $\mathrm{H}^{1}(g, G): \mathrm{H}^{1}(Z, G) \rightarrow \mathrm{H}^{1}(Y, G)$ est un isomorphisme pour tout L-groupe fini $G$;

(ii) pour tout entier $m$ à support dans $\mathrm{L}$, et tout $\mathbf{Z} / m \mathbf{Z}$-faisceau lisse $\mathscr{L}$ sur $Z$, se factorisant par $\pi_{1}^{\mathrm{L}}(Z)$, les morphismes

$$
\mathrm{H}^{i}(g): \mathrm{H}^{i}(Z, \mathscr{L}) \longrightarrow \mathrm{H}^{i}\left(Y, \mathscr{L}_{\mid Y}\right)
$$

sont des isomorphismes.

Par la suite, les faisceaux lisses du (ii) seront simplement appelés faisceaux lisses L-monodromiques.

TOME $131-2003-\mathrm{N}^{\mathrm{O}} 1$ 
Compte-tenu du 4.4, on peut supposer que pour toute générisation géométrique $t \rightarrow s$ de points de $S$, la condition (i) (avec $\mathrm{L}=\mathrm{L}_{S}$ ) est satisfaite pour les morphismes

$$
X_{s} \longrightarrow X_{S_{(s)}\langle t\rangle} \stackrel{\text { déf }}{=} X_{(s)}\langle t\rangle \quad \text { et } \quad X_{t} \longrightarrow X_{(s)}\langle t\rangle .
$$

Il nous reste à vérifier l'énoncé de cohomologie abélienne.

D'après 3.4, on peut supposer le morphisme $f$ universellement localement acyclique (pour les coefficients abéliens). Soient $t \rightarrow s$ une spécialisation de points géométriques et $\mathscr{L}$ un faisceau lisse $\mathrm{L}$-monodromique sur $X_{(s)}\langle t\rangle$. Pour chaque entier $i$, le morphisme $\mathrm{H}^{i}\left(X_{(s)}\langle t\rangle, \mathscr{L}\right) \rightarrow \mathrm{H}^{i}\left(X_{t}, \mathscr{L}\right)$ est un isomorphisme. Ainsi, pour démontrer corollaire, il suffit de le vérifier le critère (ii), pour les indices $i \leq 2 d=r$, où $d$ est un majorant de la dimension des fibres de $f$. Quitte à rétrécir $S$, et faire un changement de base inoffensif, on peut supposer qu'il existe un hyper-recouvrement tronqué $\varepsilon: X_{\bullet \leq r+1} \rightarrow X$, qui soit le complémentaire d'un diviseur à croisement normaux relatifs dans un schéma simplicial tronqué propre et lisse sur $S$ ( $c f .2 .6$ et la remarque qui suit). Nous allons voir que la conclusion du corollaire est valable sur $S$. Pour $\alpha \in\{0, \ldots, r+1\}$, notons $f_{\alpha}$ le morphisme lisse $X_{\alpha} \rightarrow S$ déduit de $f$, et $f_{(s)}\langle t\rangle_{\alpha}$ les morphismes déduits par changement de base $S_{(s)}\langle t\rangle \rightarrow S$. Il ne nous reste plus qu'à montrer que les morphismes $\mathrm{H}^{i}\left(X_{(s)}\langle t\rangle, \mathscr{L}\right) \rightarrow \mathrm{H}^{i}\left(X_{s}, \mathscr{L}\right)$ sont des isomorphismes pour $0 \leq i \leq r$.

Pour chaque indice $\alpha$, le faisceau $\mathscr{L}_{X_{\alpha(s)}\langle t\rangle}$ est par hypothèse modérément ramifié à l'infini si bien que pour chaque $i \in \mathbf{N}$, la formation des $S_{(s)}\langle t\rangle$-faisceaux $\mathrm{R}^{i} f_{(s)}\langle t\rangle_{\alpha_{*}} \mathscr{L}_{\mid X_{(s)}\langle t\rangle_{\alpha}}$ est compatible au changement de base (cf. [19], 1.3.3). La conclusion résulte maintenant $\mathrm{du}$ fait que, par descente cohomologique, on a l'égalité $\tau_{\leq r} \mathrm{R} f_{(s)}\langle t\rangle_{*} \mathscr{L}=\tau_{\leq r} \mathrm{R}\left(f_{(s)}\langle t\rangle_{\bullet \leq r+1}\right)_{*} \mathscr{L}_{\bullet}$.

On peut aussi montrer que les flèches $X_{s} \rightarrow X \times{ }_{S} S_{(s)} \leftarrow X_{t}$ induisent des Ł-équivalences.

Pour étudier l'extension, en caractéristique positive, du corollaire précédent au cas où $\mathrm{L}=\{2,3, \ldots\}$, il est naturel de s'intéresser à la question suivante. Soit $X \rightarrow S$ un morphisme lisse, $\ell$ un nombre premier inversible sur $S$; existe-t-il un ouvert dense de $S$ tel que pour tout $\mathbf{F}_{\ell}$-faisceau lisse $\mathscr{F}$ sur $X$, et tout point géométrique $u$ de $U$, les morphismes $\mathrm{H}^{i}\left(X \times{ }_{S} U_{(u)}, \mathscr{F}\right) \rightarrow \mathrm{H}^{i}\left(X_{u}, \mathscr{F}\right)$ soient des isomorphismes? Il n'en est rien comme le montre l'exemple suivant que LEI Fu a aimablement communiqué à l'auteur.

Soient $k$ la clôture algébrique d'un corps fini, $\ell$ un nombre premier inversible sur $k$, et $q: X=\mathbf{A}_{k}^{1} \times{ }_{k} \mathbf{A}_{k}^{1} \rightarrow S=\mathbf{A}_{k}^{1}$ la seconde projection. Notons $p$ la première projection. Supposons un instant qu'il existe un ouvert non vide $U$ de $S$ tel que la formation de $\mathrm{R} q_{*} \mathscr{F}$ commute aux changements de bases $\bar{u} \rightarrow U$, avec $\bar{u}$ localisé en un point fermé, pour tout $\mathbf{F}_{\ell}$-faisceau lisse $\mathscr{F}$. Par $t$-exactitude de la transformation de Fourier sur $S$, on sait que si $\mathscr{L}$ désigne un 


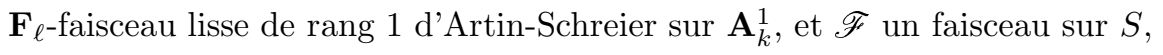

$$
\mathrm{R} q_{!}\left(p^{*} \mathscr{F} \otimes \mathscr{L}(\langle,\rangle)\right)=\mathrm{R} q_{*}\left(p^{*} \mathscr{F} \otimes \mathscr{L}(\langle,\rangle)\right) .
$$

Si maintenant $a$ est un point géométrique de $U$, on a

$$
\mathrm{H}_{c}^{2}\left(\mathbf{A}_{k}^{1}, \mathscr{F} \otimes \mathscr{L}(x a)\right)=\mathrm{H}^{2}\left(\mathbf{A}_{k}^{1}, \mathscr{F} \otimes \mathscr{L}(x a)\right) .
$$

Si l'on pose $\mathscr{F}=\mathscr{L}(-x a)$, le terme de gauche est non nul (par dualité) alors que celui de droite est trivial. Absurde.

Proposition 4.7 (formule de Künneth). - Soit $k$ un corps séparablement clos d'exposant caractéristique $p \geq 1$. Soient $X, Y$ deux $k$-schémas connexes de type fini, et $(x, y) \in X(k) \times Y(k)$. Le morphisme

$$
\pi_{1}^{p \prime}\left(X \times_{k} Y,(x, y)\right) \longrightarrow \pi_{1}^{p \prime}(X, x) \times \pi_{1}^{p \prime}(Y, y)
$$

défini par covariance $d u \pi_{1}^{p \prime}$, est un isomorphisme.

Il est bien connu que cela est faux pour le $\pi_{1}$ entier.

Pour la commodité du lecteur, nous rappelons comment déduire la formule de Künneth des théorèmes de propreté cohomologique et locale acyclicité génériques.

Démonstration. - Notons $P$ le $k$-schéma $X \times_{k} Y$ et $f$ la projection $P \rightarrow Y$. Il suffit de montrer que les morphismes $P_{y}=X \times\{y\} \rightarrow P \stackrel{f}{\rightarrow} Y$ induisent une suite exacte

$$
1 \rightarrow \pi_{1}^{p \prime}(X \times\{y\}) \longrightarrow \pi_{1}^{p \prime}(P) \longrightarrow \pi_{1}^{p^{\prime}}(Y) \rightarrow 1
$$

En effet, comme cette suite s'envoit sur la suite exacte tautologique

$$
1 \rightarrow \pi_{1}^{p \prime}(X) \longrightarrow \pi_{1}^{p \prime}(X) \times \pi_{1}^{p \prime}(Y) \longrightarrow \pi_{1}^{p \prime}(Y) \rightarrow 1,
$$

le morphisme $\pi_{1}^{p \prime}\left(X \times_{k} Y\right) \rightarrow \pi_{1}^{p \prime}(X) \times \pi_{1}^{p \prime}(Y)$ sera un isomorphisme par exactitude.

L'injectivité (à gauche) est automatique car le composé de

$$
\pi_{1}^{p^{\prime}}(X \times\{y\}) \longrightarrow \pi_{1}^{p^{\prime}}(P)
$$

avec $\pi_{1}^{p^{\prime}}(P) \rightarrow \pi_{1}^{p^{\prime}}(X)$ est un isomorphisme. La surjectivité de $\pi_{1}^{p^{\prime}}(P) \rightarrow \pi_{1}^{p \prime}(Y)$ se traduit de la façon suivante : pour tout revêtement étale connexe galoisien d'ordre premier à $p, Y^{\prime} \rightarrow Y$, le schéma $X \times_{k} Y^{\prime}=P \times_{Y} Y^{\prime}$ est connexe; cela résulte du fait que les fibres géométriques de $f$ sont connexes (i.e. $f$ est 0 -acyclique, $c f$. [3, déf. 1.1 ii et cor. 1.16 avec $n=0]$ ), et n'utilise pas l'hypothèse que $p$ est inversible sur $k$. Pour établir l'exactitude au centre, nous allons vérifier, comme dans $[15,1.2]$, que tout $p^{\prime}$-revêtement connexe de $P$ scindé sur $P_{y}$ est image inverse d'un $p^{\prime}$-revêtement de $Y$. Soit $P^{\prime}$ un tel revêtement, de degré $d$, et

TOME $131-2003-\mathrm{N}^{\mathrm{O}} 1$ 
notons $\mathrm{h}_{P^{\prime}}=\operatorname{Hom}_{P}\left(-, P^{\prime}\right)$ le faisceau de Yoneda associé, localement constant constructible. Nous démontrerons un peu plus bas le résultat suivant :

$\left\{\right.$ le faisceau $f_{*} \mathrm{~h}_{P^{\prime}}$ sur $Y$ est localement constant constructible, et de \{formation compatible aux changements de bases (relativement à $Y$ ).

Ainsi, l'espace algébrique étalé associé à ce faisceau est un revêtement étale $Y^{\prime} \rightarrow Y$. La flèche d'adjonction au niveau des faisceaux $f^{*} f_{*} \mathrm{~h}_{P^{\prime}} \rightarrow \mathrm{h}_{P^{\prime}}$ correspond $^{(5)}$ à un morphisme de $P$-schémas étales : $Y^{\prime} \times_{Y} P \rightarrow P^{\prime}$. Regardons la fibre en $z=(x, y)$ de ce morphisme de faisceaux : le terme de gauche est

$$
\left(\mathrm{h}_{Y^{\prime}}(y)=\right) f_{*} \mathrm{~h}_{P^{\prime}}(y)=\mathrm{h}_{P_{y}^{\prime}}\left(P_{y}\right)
$$

par commutation au changement de base, tandis que le terme de droite est $\mathrm{h}_{P^{\prime}}(z)=f^{-1}(z)$, de cardinal $d$. Géométriquement, cette flèche n'est autre que la restriction des sections. Le revêtement $P^{\prime} / P$ étant scindé au-dessus de $P_{y}, \mathrm{~h}_{Y^{\prime}}(y)$, et par conséquent le schéma $Y^{\prime}$, sont non vides si bien que le morphisme $g: P \times_{Y} Y^{\prime} \rightarrow P^{\prime}$ est un morphisme entre revêtements non vides de $P$; le schéma $P^{\prime}$ étant connexe, le morphisme $g$ est un revêtement étale. Il en résulte que

$$
\operatorname{deg}\left(Y^{\prime} \times_{Y} P / P\right)=\operatorname{deg}\left(Y^{\prime} / Y\right) \geq \operatorname{deg}\left(P^{\prime} / P\right)=d .
$$

D'un autre côté,

$$
\operatorname{deg}\left(Y^{\prime} / Y\right)=\sharp \mathrm{h}_{Y^{\prime}}(y)=\sharp \mathrm{h}_{P_{y}^{\prime}}\left(P_{y}\right) \leq d .
$$

Finalement $\operatorname{deg}(g)=1$ et $g$ est un isomorphisme. Ainsi, sous nos hypothèses, $P^{\prime}$ provient bien de la base; il reste à montrer que le revêtement $Y^{\prime} / Y$ dont il est déduit est d'ordre premier à $p$. On peut supposer par fonctorialité que $P^{\prime} / P$ est galoisien de groupe un $p^{\prime}$-groupe $G$ (scindé, donc totalement décomposé, sur $\left.P_{y}\right)$. La surjectivité du morphisme $\pi_{1}(P) \rightarrow \pi_{1}(Y)$ se traduit en la pleine fidélité du foncteur $\operatorname{RevÉt}(Y) \rightarrow \operatorname{RevÉt}(P)$. Dans notre cas, cela implique l'égalité $\operatorname{Aut}_{Y}\left(Y^{\prime}\right)=\operatorname{Aut}_{P}\left(P^{\prime}\right)=G$, si bien que $Y^{\prime} / Y$ est lui-aussi galoisien de groupe $G$.

Il nous faut maintenant justifier l'hypothèse $(\mathrm{H})$. Il existe un revêtement galoisien $P^{\prime \prime}$ de groupe $G$ de $P$, et un sous-groupe $H$ de $G$ tel que $P^{\prime}=P^{\prime \prime} / H$. D'après $[24,1.16$ (iii)], pour démontrer la commutation au changement de base, il suffit de vérifier que les couples $(f, \operatorname{Tors}(G))$ et $(f, \operatorname{Tors}(H))$ sont cohomologiquement propres (relativement à $Y$ ). Or, il résulte de 2.4 que cela est vrai pour tout $p^{\prime}$-groupe (constant), $f$ se déduisant du morphisme $X \rightarrow \operatorname{Spec}(k)$ par changement de base. Une fois la commutation au changement de base acquise, la constructibilité est automatique, d'après le lemme suivant :

(5) Rappelons que si un faisceau étale $\mathscr{F}$ sur un schéma $S$ est représenté par un $S$-schéma $T$, pour tout morphisme $c: S^{\prime} \rightarrow S$, le $S^{\prime}$-faisceau $c^{*} \mathscr{F}$ est représentable par le schéma $S^{\prime} \times{ }_{S} T$.

BULletin DE LA SOCIÉtÉ MATHÉMATIQUE DE FRANCE 
LEMme 4.8. - Soient $\alpha: A \rightarrow B$ un morphisme de type fini entre schémas noethériens et $\mathscr{F}$ un faisceau d'ensembles constructible tel que $(\alpha, \mathscr{F})$ soit cohomologiquement propre. Alors, le faisceau $\alpha_{*} \mathscr{F}$ sur $B$ est constructible.

Démonstration. - En effet, par constructibilité générique, il existe un ouvert $U$ de $B$ tel que $\left(\alpha_{*} \mathscr{F}\right)_{\mid U}$ soit constructible. Notons $i: Z \hookrightarrow B$ l'immersion fermée du complémentaire réduit de $U$ dans $B$. Par hypothèse, le faisceau $i^{*} \alpha_{*} \mathscr{F}$ est isomorphe à l'image directe de $\mathscr{F}_{\mid A_{Z}}$ par $\alpha_{Z}: A_{Z}=A \times_{B} Z \rightarrow Z$. Comme $\mathscr{F}_{\mid A_{Z}}$ est constructible et que $\left(\alpha_{Z}, \mathscr{F}_{\mid A_{Z}}\right)$ est cohomologiquement propre, on conclut par récurrence noethérienne.

Il ne reste plus qu'à montrer que notre faisceau $f_{*} \mathrm{~h}_{P^{\prime}}$ est localement constant. D'après la remarque suivant 4.2 , compte-tenu de la propreté cohomologique, il suffit de montrer que si $I$ est un ensemble à deux éléments, $(f, I)$ est localement acyclique. Or il résulte de 3.3, a) (i) que $(X \rightarrow \operatorname{Spec}(k), I)$ l'est universellement, d'où le résultat.

Corollaire 4.9. - Soit $k$ un corps algébriquement clos de caractéristique $p$. Soient $X$ et $Y$ deux $k$-schémas de type fini. Le morphisme

$$
\left(X \times_{k} Y\right)_{e ́ t}^{p \prime} \longrightarrow X_{e ́ t}^{p \prime} \times Y_{e ́ t}^{p \prime}
$$

est une Ł-équivalence.

Démonstration. - Il suffit, comme dans 4.6, de vérifier deux conditions sur cette flèche : qu'elle induit des isomorphismes d'une part sur les $\mathrm{H}^{1}$ non abéliens à valeurs dans les $p^{\prime}$-groupes, et sur tous les $\mathrm{H}^{i}$ à valeurs dans des $\mathbf{F}_{\ell}$-faisceaux lisses $p^{\prime}$-monodromiques $(\ell \neq p)$ d'autre part. Compte-tenu des isomorphismes

$$
\mathrm{H}^{1}\left(\left(X \times_{k} Y\right)_{\text {ét }}^{p^{\prime}}, G\right) \cong \mathrm{H}_{\text {ét }}^{1}\left(X \times_{k} Y, G\right) \cong \operatorname{Hom}\left(\pi_{1}^{p \prime}\left(X \times_{k} Y\right), G\right)
$$

pour tout $p^{\prime}$-groupe $G$, la première condition résulte de la formule de Künneth que nous venons de démontrer et de son analogue topologique pour le produit $X_{\text {ét }}^{p \prime} \times Y_{\text {ét }}^{p \prime}$. Pour vérifier la seconde condition, remarquons que dans le cas particulier où $\mathcal{L}$ est un $\mathbf{F}_{\ell}$-faisceau lisse $p^{\prime}$-monodromique sur $X_{\text {ét }}^{p \prime} \times Y_{\text {ét }}^{p \prime}$, produit externe de deux faisceaux sur $X_{\text {ét }}^{p \prime}$ et $Y_{\text {ét }}^{p \prime}$, l'isomorphisme désiré (pour chaque $i \in \mathbf{N}$ ) n'est autre que la formule de Künneth abélienne de [8]

$$
\mathrm{R} \Gamma\left(X \times_{k} Y, \mathscr{F}_{X} \otimes \mathscr{F}_{Y}\right) \stackrel{\sim}{\longrightarrow} \mathrm{R} \Gamma\left(X, \mathscr{F}_{X}\right) \otimes^{\mathbb{L}} \mathrm{R} \Gamma\left(Y, \mathscr{F}_{Y}\right) .
$$

Remarquons maintenant que pour tout couple de groupes finis $G$ et $G^{\prime}$, les $\overline{\mathbf{F}}_{\ell}\left[G \times G^{\prime}\right]$-modules simples sont des produits externes de représentations irréductibles de $G$ et $G^{\prime}$. Soient $r \in \mathbf{N}$ et $\mathcal{L}$ un $\mathbf{F}_{\ell^{-}}$-faisceau lisse $p^{\prime}$-monodromique $\operatorname{sur} X_{\text {ét }}^{p \prime} \times Y_{\text {ét }}^{p \prime} ;$ posons $\mathcal{L}_{r}=\mathcal{L} \otimes_{\mathbf{F}_{\ell}} \mathbf{F}_{\ell^{r}}$. Pour $r \gg 0, \mathcal{L}_{r}$ est somme directe de produits externes de $\mathbf{F}_{\ell^{r}}$-faisceaux lisses, si bien que la seconde condition est satisfaite pour ce faisceau. Comme le faisceau $\mathcal{L}$ se plonge dans $\mathcal{L}_{r}$, une récurrence sur $i$ donne l'isomorphisme des $\mathrm{H}^{i}$ pour tout $i$.

TOME $131-2003-\mathrm{N}^{\mathrm{O}} 1$ 
THÉORÈmE 4.10. - Soient $k$ un corps algébriquement clos d'exposant caractéristique $p \geq 1, X$ un $k$-sous-schéma connexe d'un espace projectif $\mathbf{P}_{k}$ sur $k$. Supposons $X$ régulier de dimension $\geq 3$. Alors, si $Y$ une section hyperplane générale de $X$ on $a$ :

- le schéma $Y$ est connexe,

- pour tout point géométrique y de $Y$, le morphisme canonique $\pi_{1}^{p \prime}(Y, y) \rightarrow$ $\pi_{1}^{p \prime}(X, y)$ est un isomorphisme.

Démonstration. — L'énoncé se trouve dans [23] avec l'hypothèse supplémentaire que la caractéristique est nulle. Cette hypothèse était nécessaire pour pouvoir utiliser le théorème 2.2. Celui-ci étant maintenant démontré en toute généralité, la même démonstration donne ce théorème, à condition bien entendu de se restreindre aux $p^{\prime}$-groupes.

De même, utilisant le théorème de Michèle Raynaud sur les surfaces, on trouve :

THÉORÈmE 4.11. - Soient $k$ un corps séparablement clos d'exposant caractéristique $p \geq 1$ et $X$ un $k$-schéma algébrique. Alors le groupe $\pi_{1}^{p^{\prime}}(X)$ est topologiquement de présentation finie.

Démonstration. - On peut supposer $k$ algébriquement clos. Utilisant des altérations à la place de la résolution des singularités, on peut se ramener facilement par descente ( $c f$. [22]) au cas d'un schéma affine lisse. Utilisant le théorème de Lefschetz sur les sections hyperplanes, on se ramène au cas où $X$ est une surface, traité dans loc. cit.

\section{Discussion du cas modéré}

Les résultats de cette partie utilisent la géométrie logarithmique; nous renvoyons le lecteur à [20]. Un exposé plus systématique de la théorie de la spécialisation du groupe fondamental logarithmique sera donné dans un prochain article d'Isabelle Vidal.

Soient $k$ un corps algébriquement clos d'exposant caractéristique $p \geq 1$, $X$ un schéma régulier connexe, propre sur $k$ et $U \hookrightarrow X$ le complémentaire d'un diviseur à croisements normaux $Y$. Nous dirons que $X$ est une bonne compactification de $U$. Soit $u$ un point géométrique de $U$; notons $\pi_{1}^{t}\left(U_{/ X}, u\right)$ le quotient de $\pi_{1}(U, u)$ classifiant les revêtements de $U$ modérément ramifiés le long de $Y=X-U$; rappelons qu'il s'agit des revêtements de $U$ dont les restrictions (après normalisation) aux localisés de $X$ en les points génériques de $Y$ sont modérés, au sens valuatif classique. Il revient au même de dire que le faisceau lisse $\mathscr{L}(V / U)$ représenté par $V$ sur $U$ est modérément ramifié en ces points. 
Rappelons que le groupe fondamental modéré d'un $k$-schéma pointé connexe $U$ possédant une bonne compactification ne dépend pas du choix de celle-ci. Soient en effet $j_{1}: U \hookrightarrow X_{1}$ et $j_{2}: U \hookrightarrow X_{2}$ deux telles compactifications, $\bar{\eta}$ un point générique géométrique de $U$ et $V \rightarrow U$ un revêtement modéré de $U$ relativement à $X_{1}$ (i.e. le long de $\left.Y_{1}=X_{1}-U\right)$; on souhaite montrer qu'il est modéré le long de $Y_{2}=X_{2}-U$. Soit $Z$ une compactification de $U$ dominant $X_{1}$ et $X_{2}$ (quitte à la normaliser, on peut supposer $Z$ normal). Soit $x \in Y_{2}$ un point de codimension 1. Le schéma $Z$ étant intègre, séparé, et propre sur $X_{2}$, il résulte de EGA II 7.3.1 que $Z \times_{X_{2}} \operatorname{Spec}\left(\mathscr{O}_{X_{2}, x}\right) \rightarrow \operatorname{Spec}\left(\mathscr{O}_{X_{2}, x}\right)$ est un isomorphisme (c'est un isomorphisme générique sur un trait). Ainsi, il suffit de montrer que $V$ est modérément ramifié relativement à $Z$, en les points de codimension 1, c'est-à-dire que les $p$-Sylow des groupes d'inertie locale en ces points agissent trivialement sur $\mathscr{L}(V / U)_{\bar{\eta}}$. Soit $\bar{z}$ un point géométrique de $Z$ localisé en un point maximal de $Y=Z-U$ et notons $\bar{x}_{1}$ son image dans $X_{1}$. Il résulte du lemme d'Abhyankar et du théorème de pureté de Zariski-Nagata (le schéma $X_{1}$ est régulier), que les $p$-Sylow du groupe $\pi_{1}\left(X_{1}\left(\bar{x}_{1}\right) \times_{X_{1}} U, \bar{\eta}\right)$ agissent trivialement sur $\mathscr{L}(V / U)_{\bar{\eta}}$. Comme l'action de $\pi_{1}\left(Z(\bar{z}) \times_{Z} U, \bar{\eta}\right)$ se factorise par l'action précédente, son $p$-Sylow agit trivialement. Le résultat en découle. (La démonstration qui précède est due à Michel Raynaud.)

ThÉORÈme 5.1. - Soient $k$ un corps séparablement clos, $(U, u)$ et $(V, v)$ deux $k$-schémas pointés connexes ayant de bonnes compactifications. Alors, le morphisme $\pi_{1}^{t}\left(U \times_{k} V, u \times v\right) \rightarrow \pi_{1}^{t}(U, u) \times \pi_{1}^{t}(V, v)$ est un isomorphisme

Le point clé est l'interprétation logarithmique que l'on peut donner du groupe fondamental modéré dans le cas d'une bonne compactification : d'après un théorème de K. Fujiwara et K. Kato [10] (voir aussi $[20,7.6]$ ), $\pi_{1}^{t}\left(U_{/ X}, u\right.$ ) s'identifie à $\pi_{1}^{\log }(X, u)$, le groupe fondamental logarithmique de $X$ (pour la topologie Kummer-étale), muni de la log-structure évidente. Ainsi, la formule à démontrer devient l'analogue logarithmique de la formule de Künneth pour les schémas propres sur $k$ démontrée par A. Grothendieck dans [15, 1.7]. La démonstration de loc. cit. se recopie mot à mot à condition de vérifier l'analogue de loc. cit. 1.2 sur la factorisation de Stein. C'est l'objet de la proposition suivante :

Proposition 5.2 (I. Vidal). — Soient $\underline{U} \hookrightarrow \underline{X}$ et $\underline{V} \hookrightarrow \underline{Y}$ de bonnes compactifications, et $R \rightarrow P \stackrel{\text { déf }}{=} X \times_{k} Y$ un revêtement Kummer-étale connexe où $\underline{X}$ et $\underline{Y}$ sont munis des log-structures évidentes. Soit $\underline{R} \rightarrow \underline{Y}^{\prime} \rightarrow \underline{Y}$ la factorisation de Stein du morphisme (propre) sous-jacent. Alors $\underline{Y}^{\prime} / \underline{Y}$ est étale au-dessus de $V$ et modérément ramifié le long de $\underline{Y}-V$.

Ainsi, par Fujiwara-Kato, on peut munir $\underline{Y}^{\prime}$ d'une log-structure qui fasse de $Y^{\prime} \rightarrow Y$ un revêtement Kummer-étale.

TOME $131-2003-\mathrm{N}^{\mathrm{O}} 1$ 
Démonstration. - Le morphisme $R_{V}=R \times_{Y} V \rightarrow V$ est propre et log-lisse. Les fibres log-géométriques sont donc log-régulières, et en particulier réduites, et s'identifient aux fibres géométriques car la log-structure de $V$ est triviale. Finalement, $\underline{R}_{V} \rightarrow \underline{V}$ est séparable et $V^{\prime}=\underline{Y}^{\prime} \times_{Y} V \rightarrow V$, qui en est la factorisation de Stein, est étale. Il nous reste à montrer que l'extension correspondante est modérée le long de $D_{V}=\underline{Y}-V$; pour cela, on peut remplacer $\underline{Y}$ par son hensélisé strict en un point générique de $D_{V}$, noté $\underline{S}$. On remplace aussi $R \rightarrow P$ par une composante connexe de $R \times_{Y} S$, qui est un revêtement connexe de $X \times_{k} S$.

Le schéma $\underline{S}^{\prime}=\underline{Y}^{\prime} \times \underline{Y} \underline{S}$ est fini sur $\underline{S}$; il est de plus connexe (image d'un connexe) et normal (car $\underline{R}_{\underline{S}}$, noté maintenant $\underline{R}$, l'est). C'est donc un trait, fini et génériquement étale sur $\underline{S}$. Soit $\underline{S}_{t}$ l'extension modérée maximale de $\underline{S}$ dans $\underline{S}^{\prime}$; on veut montrer que $\underline{S}_{t}=\underline{S}^{\prime}$. Le revêtement $\underline{S}_{t} / \underline{S}$ est étale modéré; le trait $\underline{S}_{t}$, muni de la log-structure naturelle, est donc un revêtement Kummerétale de $S$. Le morphisme $R \rightarrow S_{t}$ est donc log-lisse et $\underline{R} \rightarrow \underline{S}^{\prime} \rightarrow \underline{S}_{t}$ en est la factorisation de Stein. Supposons l'extension totalement sauvage $\underline{S}^{\prime} / \underline{S}_{t}$ non triviale, et montrons que $R$ n'est pas connexe. On peut donc supposer $S=S_{t}$ dans la suite. Notons $R^{\prime}=R \times{ }_{S} S^{\prime}$ le produit comme log-schémas fs, et $\eta$ (resp. $\eta^{\prime}$ ) le point générique de $S$ (resp. $S^{\prime}$ ). Comme $\eta^{\prime} \times_{\eta} \eta^{\prime}$ n'est pas connexe, la fibre générique de $\underline{R}^{\prime}$ ne l'est pas. Le log-schéma $R^{\prime}$ étant log-régulier (car log-lisse sur $S^{\prime} \log$-régulier), $\underline{R}^{\prime}$ est normal si bien que $\pi_{0}\left(\underline{R}^{\prime}\right)=\pi_{0}\left(\underline{R}_{\eta^{\prime}}^{\prime}\right)$, l'ouvert $\underline{R}_{\eta^{\prime}}^{\prime}$ étant dense dans $\underline{R}^{\prime}$. Finalement, on voit que $\underline{R}^{\prime}$ n'est pas connexe; il en est donc de même de sa log-fibre spéciale $R_{s^{\prime}}^{\prime}=R_{s^{\prime}}$. Cependant, $R_{s^{\prime}} \rightarrow R_{s}$ est un homéomorphisme universel de Kummer car $s^{\prime} \rightarrow s$ l'est, alors que $R_{s}$ est connexe comme on le déduit de la connexité de $R$ par la factorisation de Stein. Absurde.

Cette proposition entraîne, comme dans [15, 1.3-1.4], l'exactitude de la suite

$$
\pi_{1}^{\log }(X) \longrightarrow \pi_{1}^{\log }\left(X \times_{k} Y\right) \longrightarrow \pi_{1}^{\log }(Y) \rightarrow 1,
$$

dont on déduit la formule de Künneth (5.1), comme en 4.7.

Remarque 5.3. - On peut aussi montrer que si est $k$ un corps séparablement clos, $(U, u)$ un $k$-schéma pointé connexe ayant une bonne compactification, et $K / k$ un sur-corps séparablement clos de $k$, le morphisme canonique $\pi_{1}^{t}\left(U \times_{k} K, u\right) \rightarrow \pi_{1}^{t}(U, u)$ est un isomorphisme.

Indiquons rapidement l'idée, due à O. Gabber. Soient $X$ une bonne compactification de $U$ et $G$ un groupe fini. On a un morphisme canonique de topos $\alpha: X_{K \text { ét }} \rightarrow X_{\text {ét }}$, où $X_{K \text { ét }}$ est le topos Kummer-étale associé à la log-structure évidente sur $X$, définie par $X-U$. La remarque résulte du théorème de changement de base propre pour les champs ind-finis appliqué au morphisme propre de schémas usuels $X \rightarrow \operatorname{Spec}(k)$ et aux champs $\alpha_{*}\left(\operatorname{Tors}_{K}\right.$ ét $\left.(G)\right)$ pour $G$ variable. 


\section{BIBLIOGRAPHIE}

[1] Artin (M.) - Changement de base pour un morphisme propre, in Théorie des topos et cohomologie étale des schémas [17], exposé XII.

[2] _ Faisceaux constructibles. Cohomologie d'une courbe algébrique, in Théorie des topos et cohomologie étale des schémas [17], exposé IX.

[3] _ Morphismes acycliques, in Théorie des topos et cohomologie étale des schémas [17], exposé XV.

[4] Artin (M.) \& Mazur (B.) - Étale homotopy, Springer-Verlag, Berlin, 1969.

[5] Bosch (S.), Lütkebohmert (W.) \& Raynaud (M.) - Néron models, Springer-Verlag, Berlin, 1990.

[6] Deligne (P.) - La formule de dualité globale, in Théorie des topos et cohomologie étale des schémas [17], exposé XVIII.

[7] _ Théorie de Hodge III, Publ. Math. Inst. Hautes Études Sci., t. 44 (1974), pp. 5-77.

[8] Cohomologie étale, Lecture Notes in Math., vol.569, SpringerVerlag, 1977, avec la collaboration de J.-F. Boutot, A. Grothendieck, L. Illusie et J.-L. Verdier.

[9] Friedlander (E.M.) - Étale homotopy of simplicial schemes, Princeton University Press, Princeton, N.J., 1982.

[10] Fujiwara (K.) \& Kato (K.) - Logarithmic étale topology theory, Prépublication.

[11] GabBer (O.) - Lettre à l'auteur, octobre 2001.

[12] Lettre à l'auteur, décembre 2001.

[13] Giraud (J.) - Cohomologie non abélienne, Grundlehren der math. Wissen., vol. 179, Springer-Verlag, Berlin, 1971.

[14] Grothendieck (A.) - Revêtements étales et groupe fondamental, Séminaire de Géométrie Algébrique du Bois-Marie 1960-1961 (SGA1), Lecture Notes in Math., vol. 224, Springer-Verlag, 1971.

[15] - Théorie de la spécialisation du groupe fondamental, in Revêtements étales et groupe fondamental [14], exposé X.

[16] Groupes de monodromie en géométrie algébrique, Séminaire de Géométrie Algébrique du Bois-Marie 1967-1969 (SGA7 I \& II), Lecture Notes in Math., vol. 288 \& 340, Springer-Verlag, 1972.

[17] _ Théorie des topos et cohomologie étale des schémas, Séminaire de Géométrie Algébrique du Bois-Marie 1963-1964 (SGA4), Lecture Notes in Math., vol. 269-270 \& 305, Springer-Verlag, 1972-1973.

[18] Illusie (L.) - Complexe cotangent et déformations, Lecture Notes in Math., vol. 239 \& 283, Springer-Verlag, Berlin, 1971-1972.

[19] _ Appendice à [Th. finitude], in Cohomologie étale [8], 1977.

TOME $131-2003-\mathrm{N}^{\mathrm{O}} 1$ 
[20] _ An overview of the work of K. Fujiwara, K. Kato, and C. Nakayama on logarithmic étale cohomology, in Périodes p-adiques II (Berthelot (P.), Fontaine (J.-M.), Illusie (L.), Kato (K.) \& Rapoport (M.), éds.), Astérisque, vol. 279, Soc. Math. France, Paris, 2002.

[21] DE Jong (A.J.) - Smoothness, semi-stability and alterations, Publ. Math. Inst. Hautes Études Sci., t. 83 (1996), pp. 51-93.

[22] Raynaud (M.) - Propriétés de finitude du groupe fondamental, in Groupes de monodromie en géométrie algébrique [16], exposé II.

[23] _ Théorèmes de Lefschetz en cohomologie des faisceaux cohérents et en cohomologie étale. Application au groupe fondamental, Ann. Sci. École Norm. Sup. (4), t. 7 (1974), pp. 29-52.

[24] Raynaud (MichÈLE) - Propreté cohomologique des faisceaux d'ensembles et des faisceaux en groupes non commutatifs, in Revêtements étales et groupe fondamental [14], exposé XIII.

[25] Saint-Donat (B.) - Technique de descente cohomologique, in Théorie des topos et cohomologie étale des schémas [17], exposé v bis. 